\title{
嫦娥三号两相流体回路的地面试验验证方法 及试验结果分析
}

\author{
张红星"，苗建印，王录，莫青，刘自军，向艳超 \\ 中国空间技术研究院，北京空间飞行器总体设计部，空间热控技术北京市重点实验室，北京 100094 \\ *E-mail: redstarbenben@163.com
}

收稿日期: 2014-02-15; 接受日期: 2014-03-28

\begin{abstract}
摘要两相流体回路在我国嫦娥三号月球探测器上的应用是全球航天器热控系统设计 上的首次尝试, 两相流体回路利用月球 $g / 6$ 重力驱动, 无需电源供给, 在长达 $14 \mathrm{~d}$ 的月夜工 况下, 有效利用同位素热源解决了着陆器和巡视器内的仪器温控难题. 回路研制过程中遇 到了传热性能无法在地面得到真实模拟的技术难题, 本文以巡视器两相流体回路为例, 建 立了 $g / 6$ 重力驱动两相流体回路传热性能的地面模拟方法, 给出了地面真空热性能试验验 证结果并进行了分析.
\end{abstract}

关键词

两相流体回路

嫦娥三号

地面试验验证

\section{1 引言}

嫦娥三号月球探测器 2013 年成功在月球表面着 陆, 着陆器将在月球表面工作 12 个月, 即 12 个月球 昼夜, 巡视器工作 3 个月. 由于月球表面的温度环境 十分恶劣, 月昼时月表温度高达 $120^{\circ} \mathrm{C}$, 而月夜低至 $-180^{\circ} \mathrm{C}$. 热控分系统面临的最大挑战是如何在没有 能源供给的情况下帮助探测器度过长达 14 个地球日 的月夜, 确保巡视器和着陆器中的仪器设备处于适 宜的温度水平.

美国的月球探测器使用携带大量电池加热的方 式帮助仪器设备度过月夜. 俄罗斯的月球车则采用 “同位素热源(RHU)+密封舱内对流”的方法来控制仪 器设备的温度. 中国空间技术研究院提出了一种新 的解决方法, 设计了一种 $g / 6$ 重力驱动的两相流体回 路, 可在月球表面实现热量的高效传输. 于 2007 年 9
月, 提出了两相流体回路的方案, 2008 年完成了实验 室原理样机研制, 开展了功能验证. 2009 年 10 月通 过了方案阶段等效试验验证. 2011 年完成了初样产品 真空热性能试验及巡视器和着陆器的热平衡试验. 2012 年交付了正样飞行产品. 2013 年探测器成功着 陆并开展巡视活动, 两相流体回路正常工作, 经历了 月昼、昼夜转换、月夜等多个工况, 功能和性能完全 满足使用要求.

两相流体回路在我国月球探测器上的应用是全 球航天器热控系统设计上的首次尝试. 通过大量可 靠性与功能试验验证、设计优化, 回路可利用月球 $g / 6$ 重力驱动, 无需电源供给, 即可将舱外同位素热 源的热量引入舱内, 实现了有效的热控制. 但是, 研 制过程中曾面临的一个最艰难的技术问题就是两相 流体回路的具体传热性能无法在地面得到真实的模 拟. 两相流体回路的驱动力和阻力决定其传热能力,

引用格式: 张红星, 苗建印, 王录, 等. 嫦娥三号两相流体回路的地面试验验证方法及试验结果分析. 中国科学: 技术科学, 2014, 44: 589-596 Zhang H X, Miao J Y, Wang L, et al. Ground test method and results of closed two-phase thermosyphons for the moon exploration spacecraft Chang'E-3 (in Chinese). Sci Sin Tech, 2014, 44: 589-596, doi: 10.1360/092014-55 
驱动力和阻力受重力加速度的影响, 地球和月球表 面重力加速度的差异导致地面等效验证面临困难.

本文将主要以巡视器用流体回路为例，分析如 何通过地面试验对流体回路的性能进行验证以保证 其在月球上的正常运行, 并给出相关的地面试验验 证结果.

\section{2 两相流体回路设计及试验验证方法}

\section{1 两相流体回路设计及工作原理}

两相流体回路系统组成如图 1 所示, 巡视器的两 相流体回路设计如图 2 所示, 管壳材料不锈钢, 工质 为氨.

蒸发器从同位素热源吸收热量, 工质氨吸热蒸 发后，沿蒸气管路流到冷凝器中管路冷凝并释放热 量, 冷却后的液态氨流入储液器中, 液态工质氨流出 储液器后经过液体管路最终回流到各个蒸发器中, 继续吸热蒸发.

两相流体回路之所以能在月面运行是由于月面 具有引力, 其加速度约为 $g / 6$, 因此当一定的热量作

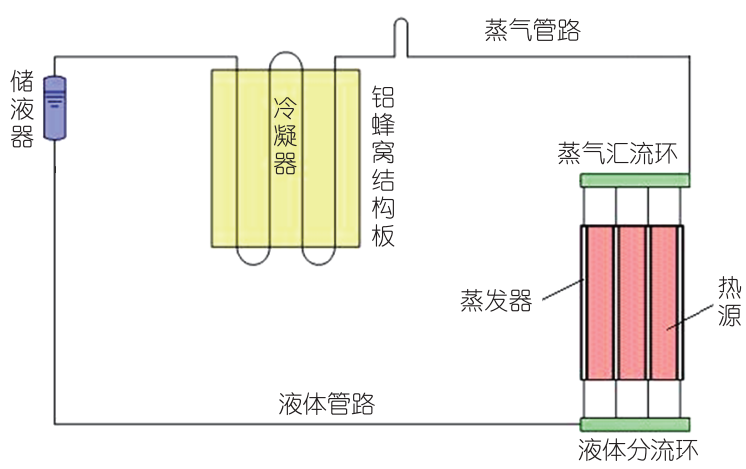

图 1 (网络版彩图)两相流体回路系统示意图

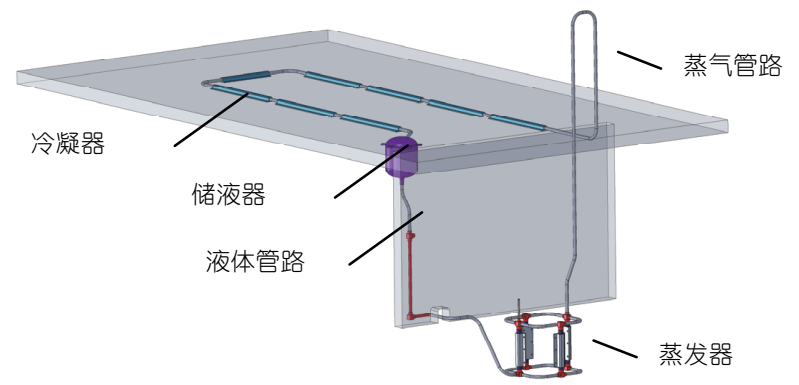

图 2 (网络版彩图)两相流体回路布局图
用在回路蒸发器上时, 蒸发器侧的工质氨将蒸发变 为气相状态, 而此时储液器侧仍然有一定液位的液 相氨工质存在, 这样在回路两侧形成了 2 个液面, 其 高度差为 $\Delta h$

由于两侧工质的密度差不同，使得回路中产生 如下式描述的驱动压力 $\Delta P_{\mathrm{d}}$ :

$$
\Delta P_{\mathrm{d}}=\left(\rho_{1}-\rho_{\mathrm{v}}\right) \Delta h_{\mathrm{m}} \frac{g}{6} .
$$

在此驱动压力下, 工质循环运行起来, 工质在回 路的各个部分的流动就会在蒸发器、蒸气管路、冷凝 管路、液体管路等部分产生如下式所示的阻力 $\Delta P_{\mathrm{z}}$ :

$$
\Delta P_{\mathrm{z}}=\Delta P_{\mathrm{e}}+\Delta P_{\mathrm{g}}+\Delta P_{\mathrm{c}}+\Delta P_{1},
$$

当回路中形成的驱动压力 $\Delta P_{\mathrm{d}}$ 与阻力 $\Delta P_{\mathrm{z}}$ 有如 下所述的匹配关系时，回路就可以稳定运行了:

$$
\Delta P_{\mathrm{d}} \geqslant \Delta P_{\mathrm{z}} .
$$

\section{2 地面模拟试验方法}

两相流体回路最终将在月面 $g / 6$ 环境下使用，而 两相流体回路产品的实验只能在地面 $1 g$ 环境下进行 各方面的性能测试，在这种条件下，可供选择的地面 模拟实验方法有如下几条.

1) 在地面人造 $g / 6$ 的环境, 进行两相流体回路 真实全模拟实验

目前在地面模拟微小重力场已经有一些手段, 例如自由落塔、飞机失重飞行等. 这些手段是能够在 地面对微小重力现象进行研究. 但能够提供的试验 时间有限，自由落塔在秒量级、飞机失重飞行在分钟 量级. 而两相流体回路的实验验证, 1 个工况就需要 数十分钟到几个小时, 因此这些手段都无法满足两 相流体回路在地面 $g / 6$ 的全模拟实验要求.

2) 在地面 $1 g$ 环境下, 进行两相流体回路的等效 模拟实验

在 $1 g$ 环境下进行地面等效模拟试验, 其原理是 将回路在地面模拟时的驱动高度差 $\Delta h_{\mathrm{e}}$ 变为月面驱 动高度差 $\Delta h_{\mathrm{m}}$ 的 $1 / 6$, 以等效模拟月面的驱动力; 但 是, 这个方法仍存在 2 个问题:

第一，只能等效模拟某一工况下的驱动高度，无 法模拟所有工况下的真实驱动高度, 因此只能采用 保守验证的方法，使除了这一等效模拟工况外其他 工况下的地面模拟驱动力小于月球实际驱动力. 
第二, 无法精确模拟 $g / 6$ 下回路的实际流动阻力, $1 \mathrm{~g}$ 下工质流动阻力会与月面有差异, 因此回路在月 面的实际传热能力可能会小于地面, 如果回路传热 能力留有一定的设计余量, 流体回路各工况未达到 其传热极限, 则地面验证有效; 若设计余量不够大, 则地面试验验证流体回路可行不代表月面也可行.

3) 在理论模型中对 $g / 6$ 进行模拟分析计算

在分析模型中对 $g / 6$ 进行模拟, 给出分析结果. 但目前即便是在地面 $1 g$ 环境下对于两相流动的准确 模拟仍较困难, 依靠目前的认知水平, 还无法准确或 量化给出 $g / 6$ 下的两相流体回路阻力, 相关的分析结 果仅可作为参考.

\section{3 重力环境对两相流动和传热的影响分析}

微重力环境下, 两相流动中由密度差导致的非 对称流动及分层流动现象减弱或消除, 流型产生和 转化的机理、相分布、以及压降和传热特性与常重力 存在差别. 根据地面试验得出的两相流压降和换热 计算的实验关联式或半试验关联式将不再适用 ${ }^{[1 \sim 4]}$.

对于两相流动本身的准确模拟还是学术界的难 题, 特别是微小重力场环境下两相流动问题, 本节主 要介绍国内外微重力场对两相流动流阻和换热影响 的理论和试验结果.

\section{1 微重力场下的两相流阻力变化}

Heppner 等人 ${ }^{[2]}$ 通过 NASA KC-135 失重飞机对 微重力下的两相流型和阻力进行了试验测试, 试验 中使用空气和水模拟两相流动, 流动通道的管内径 为 $25.4 \mathrm{~mm}$, 长径比约 20 , 水平姿态, 试验结果显示 微重力下的两相流动阻力比重力条件下高. 但是这 次试验结果存在一些问题, 流动通道的长径比过小, 而相同试验条件下的多次重复测量结果差异较大.

文献[3,4]介绍了 R114 工质在常重力和微重力场 中(通过 KC-135 失重飞行)的两相流阻阻力试验数据, 管内径 $15.8 \mathrm{~mm}$, 水平姿态, 试验中干度由 $0.05 \sim 0.9$ 变化, 液相表观速度为 $0.02 \sim 0.16 \mathrm{~m} / \mathrm{s}$, 当干度为 $0.05 \sim 0.1$ 时, 观察到了塞状流; 干度为 $0.15 \sim 0.9$ 时观 察到了环状流. 试验结果表明: 相同干度下, 微重力 场下的两相流动阻力要比常重力场高 40\%. 但文献[4] 分析认为该次试验存在诸多问题, 如压力传感器的 精度仅为 $345 \mathrm{~Pa}$, 测量的压差为 $62 \sim 1792 \mathrm{~Pa}$, 而测得
的大部分压差数据在 $345 \mathrm{~Pa}$ 左右, 且采样周期较长 $(2$ $\mathrm{s})$, 所以相关的结论可能产生一些误导.

Colin 等人 ${ }^{[5]}$ 采用相同的方法, 利用法国的“快 帆”(Caravelle)失重飞机对管径为 $6,9,11 \mathrm{~mm}$ 的管内 气液两相流的流型及阻力压降进行了试验测试, 同 样发现微重力条件下管内气液两相流的阻力压降要 大于常重力条件下的阻力压降, 并且随着管径的减 小, 阻力压降也有所升高.

在以 R12 为工质进行了微重力环境中蒸发和冷 凝的试验研究中, 1 个两相洜用于驱动氟利昂混合物 的流动, 在 NASA KC135 飞机上进行了 5 次飞行实验. 测试中, 1- $g$ 环境下的层状流和环状流流型在 0- $g$ 环境 中变成了泡状流、弹状流和环状流. 实验结果表明, 对于在直径为 $8 \mathrm{~mm}$, 质量流量为 $0.00653 \sim 0.0544$ $\mathrm{kg} / \mathrm{s}$ 的管路中, $1-\mathrm{g}$ 和 $0-\mathrm{g}$ 环境中两相流压降在统计上 没有重大差别 ${ }^{[4]}$.

一些可信度较高的微重力试验数据均是研究坚 直状态管内两相流动的阻力和换热变化. 如加拿大 Saskatchewan 大学微重力研究小组通过在 NASA KC-135 失重飞机上进行的双组份(水和空气)气液两 相流实验, 测量了在不同重力条件下坚直放置管内 气液两相流的阻力压降及当量换热系数, 其中微重 力试验持续时间为 $8 \sim 10 \mathrm{~s}$, 液相表观速度的试验范围 为 $0.1 \sim 2.5 \mathrm{~m} / \mathrm{s}$, 气相表观速度的实验范围为 $0.1 \sim 18$ $\mathrm{m} / \mathrm{s}$. 文献比较了气相表观速度为 1.5 和 $2.5 \mathrm{~m} / \mathrm{s}$ 的实 验数据, 定义气相表观速度与气液相表观速度之和 之比为横坐标, 实验结果表明: 两种重力场中, 流动 阻力都随着气相表观速度所占份额的增大而增大, 而不同气相表观速度占有份额下微重力场中的流阻 比常重力场中大 $3 \%$ 14\%, 但大部分工况下微重力引 起的阻力增大小于 $10 \%{ }^{[3,4]}$.

文献[4]总结了微重力场下的两相流理论模型的 准确性. 均相模型预测结果与实验数据的比较, 二者 的符合程度较差, 使用气液混合粘度计算, 整个干度 区域的均方根偏差约为 $28 \%$, 模型预测结果普遍较 低; 而使用液相黏度计算, 均方根偏差达 $40 \%$, 模型 预测结果则普遍较高. 微重力条件下气/液两相流摩 擦压降实验数据与 Lockhart-Martinelli-Chisholm 模型 的预测结果, 均方根偏差为 $28 \%$. 而 Friedel 模型是目 前唯一考虑了重力和表面张力等因素对两相流动摩 擦压降影响的经验模型, 但其均方根偏差仍有 $29 \%$ 可见, 由于对两相流动机理的掌握程度有限和相关 
试验关联式的缺乏, 现有模型很难给出量化的影响 分析.

综上所述, 目前微重力下的试验数据较少, 无与 本系统工质、管径、流速相近的试验数据可供借鉴, 可用于准确预测阻力的数学模型也尚未建立. 但是, 大部分飞行试验和理论分析的结果均认为：微重力 下两相流动的阻力会增大, 但目前难以通过试验或 模型预测给出阻力增大的量化结论.

\section{2 微重力场下的两相流换热变化}

文献[4]总结了微重力下两相换热的试验数据. Papell 通过失重飞机进行了微重力下两相流动沸腾换 热研究, 结果表明, 重力的减少导致流动模型由泡状 流转变为弹状流, 使微重力下换热系数比常重力下 大 $15 \%$. 而 Feldmanis 的试验表明重力对对流冷凝换 热的影响很小. Reinharts 等人在 NASA 的 KC-135 失 重飞机上使用 R12 回路进行试验, 结果表明微重力 条件下冷凝换热系数降低了 $26 \%$, 而沸腾换热系数 无明显变化. Ohta 等人在失重飞行试验中使用 R113 回路进行试验, 在内径 $8 \mathrm{~mm}$ 的坚直向上的管内沸腾 流动中, 换热系数无明显变化. 但当流速增大、加热 功率减小导致沸滕现象减弱后, 微重力下的阻力比 常重力场中要减小.

上述相互冲突的结论表明, 需要进行更多、更细 致的试验去研究微重力环境对两相换热的影响. 但 对于本系统而言, 冷凝器的最大换热热阻为辐射热 阻, 相比而言冷凝换热系数已经很大, 可以暂不考虑 重力对冷凝换热的影响.

\section{4 地面 $1 g$ 环境的等效模拟试验方法分析}

\section{1 驱动力地面等效模拟的试验方法}

采用地面等效模拟遵循 2 个原则

1) 所有工况下, 地面试验的驱动力应不大于月 面实际驱动力, 以确保地面试验是过验证;

2) 所有工况下, 在地面试验驱动力扣除月面回 路阻力增大项后仍小于月面实际驱动力的情况下, 回路应仍能正常运行. 即不能因为地面试验驱动力 过于保守而导致地面验证失败.

驱动力的地面模拟试验方法如下:

1) 为正确模拟储液器液面高度变化引起的压头 变化, 地面模拟试验中将储液器截面积增大 6 倍.

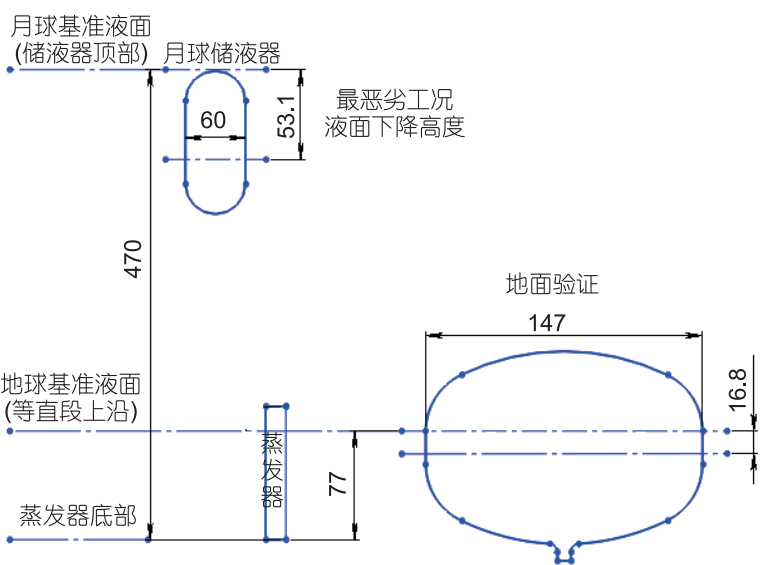

图 3 降低驱动高度等效模拟示意图

2) 降低地面的实际驱动高度, 使地面试验的驱 动力满足上述等效模拟的 2 个原则, 月面和地面上的 基准高度确定为实际的 $1 / 6$.

\section{2 阻力地面模拟的处理方法}

首先，可通过改变储液器和蒸发器相对高度的 方法模拟月球实际驱动力, 保证所有工况模拟驱动 力均不大于实际驱动力, 使地面等效验证为过验证, 测试两相流体回路各工况下的最大传热能力，通过 比较最大传热能力和实际所需传热能力, 得出回路 的实际传热能力设计余量. 还可通过地面实验件对 不同驱动高度下的传热能力进行测试，可得出满足 传热能力所需的实际驱动力, 对比可获取的最大驱 动力, 可直接得出驱动力的实际设计余量.

然后，参考中国空间技术研究院、中国科学院力 学研究所、东南大学、中山大学等单位对 $g / 6$ 下流动 阻力的国内外调研和理论建模分析, 确定 $g / 6$ 下冷凝 器内流动阻力相对地面验证时的增大百分比不超过 $30 \%$, 而冷凝器阻力占比不超过回路总阻力的 $80 \%$, 即回路总阻力增大百分比不超过 $24 \%$.

最后，在地面等效模拟月球上回路实际传热能 力的试验中, 减小地面实验中回路的驱动高度, 按阻 力的增大程度相应减小驱动力.

\section{5 试验件及试验方法}

\section{1 试验件及热控实施}

使用 2 套流体回路进行试验, 1 \#为正常充装量, 2 \#充装量较少, 其驱动高度与正常热控件高温相差 
$20 \mathrm{~mm}$, 用以模拟驱动力减少以抵消月球表面可能的 阻力增大部分.

根据巡视器的热控设计, 试验件布局示意图如 图 4 所示. 顶板的上表面先贴加热片, 后喷铈玻璃镀 银二次表面镜(OSR)替代涂层; 顶板的下表面喷有 OSR 替代涂层; 顶板与回路支架间隔热安装, 蒸发器 部件与回路支架间隔热安装. 试验件与试验支架间 隔热安装.

在试验件上布置 31 个热电偶温度传感器. 回路 上的测点位置和编号如图 5 所示.

在试验件蒸发器、储液器、蒸气液体管路、辐射 板上布置 5 个加热回路, 均采用薄膜加热器实现.

除冷凝器, 回路其他部件包裹 15 单元多层隔热 组件, 试验支架表面包覆双面镀铝聚酯膜.

\section{2 试验方法及工况}

试验在真空空间环境模拟器中进行, 通过电加 热块模拟同位素热源的发热功率. 模拟热源与蒸发 器组件贴合. 依靠流体回路的冷凝器顶板上下表面

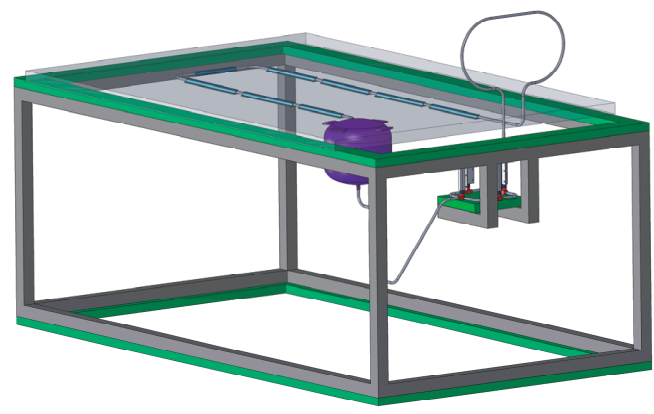

图 4 (网络版彩图)两相流体回路试验件布局示意图

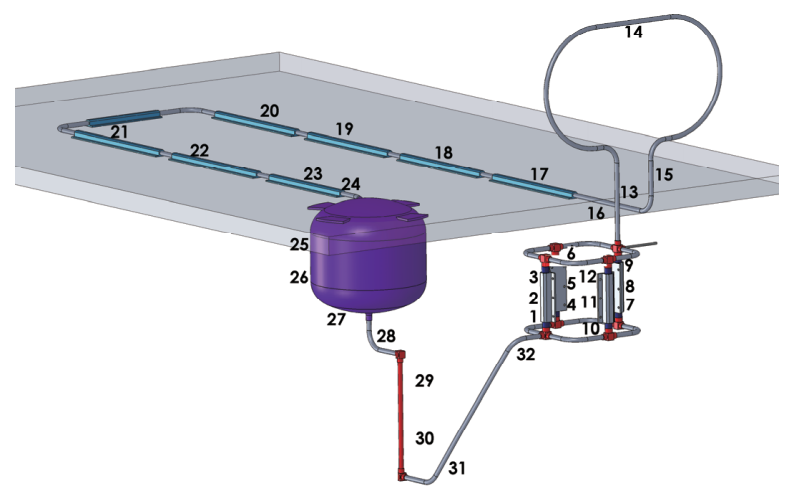

图 5 (网络版彩图)温度传感器的位置和编号
向空间热沉辐射热量. 试验过程中, 控制试验罐热沉 温度始终低于 $100 \mathrm{~K}$, 真空度优于 $1.3 \times 10^{-3} \mathrm{~Pa}$.

通过脉冲电信号控制控制阀的开关. 通过 $\mathrm{T}$ 型 热电偶测试回路特征点的温度值, 并进行实时记录 和保存.

\section{主要的试验工况包括:}

1) 稳态性能测试. 对 2 套流体回路进行稳态性 能测试, 比较各温度水平下的传热能力, 确定流体回 路的传热性能设计余量. 稳态性能测试主要是测试 回路在 $-50^{\circ} \mathrm{C},-40^{\circ} \mathrm{C},-30^{\circ} \mathrm{C}$ 下的传热能力. 具体的方 法是：通过顶板上的加热片对冷凝器加热实现对储 液器温度的控制, 使储液器的温度维持在 $-50^{\circ} \mathrm{C}$, $-40^{\circ} \mathrm{C}$ 和 $-30^{\circ} \mathrm{C}$ 温度水平. 不断增大模拟热源上的功 率, 直至回路工作温度持续上升, 无法保持平衡.

2) 动态性能测试. 首先, 对流体回路在接近传 热极限时的性能特性进行试验分析. 其次, 对试验中 稳定性进行验证.

\section{6 试验结果与分析}

\section{1 稳态传热性能与温差}

定义两相流体回路的传热温差为蒸发器上过热 最大点的温度与储液器内饱和温度之差为系统的传 热温差.

定义两流体回路的最大传热能力为传热温差不 大于 $10^{\circ} \mathrm{C}$ 下的回路传热能力.

本试验获取的两相流体回路稳态传热能力实际 上取决于 2 个因素: 第一, 回路的传热极限; 第二, 冷凝器的辐射能力. 当冷凝器的辐射能力足够大时, 试验才能获得回路的实际传热能力.

本试验件采用双面辐射散热, $-30^{\circ} \mathrm{C}$ 以下时冷凝 器辐射能力均大于回路的传热能力, 试验中为维持 回路工作温度, 对冷凝器进行了红外控温补偿加热. 因为冷凝器辐射能力足够大, 因此, 试验测得的最大 传热能力值为其实际值. 试验获取的传热能力和温 差数据如表 1 所示.

\section{表 1 流体回路稳态性能试验数据}

\begin{tabular}{cccccc}
\hline \multirow{2}{*}{ 温区 } & \multicolumn{2}{c}{ 1\#正常充装量 } & & \multicolumn{2}{c}{ 2\#留 $20 \mathrm{~mm}$ 余量 } \\
\cline { 2 - 3 } \cline { 5 - 6 } & 传热能力 & 温差 & & 传热能力 & 温差 \\
\hline$-50^{\circ} \mathrm{C}$ & $49 \mathrm{~W} @-52.4^{\circ} \mathrm{C}$ & $5.1^{\circ} \mathrm{C}$ & & $49 \mathrm{~W} @-51.7^{\circ} \mathrm{C}$ & $8.6^{\circ} \mathrm{C}$ \\
$-40^{\circ} \mathrm{C}$ & $68 \mathrm{~W} @-41.8^{\circ} \mathrm{C}$ & $4.7^{\circ} \mathrm{C}$ & & $68 \mathrm{~W} @-41.3^{\circ} \mathrm{C}$ & $6.1^{\circ} \mathrm{C}$ \\
$-30^{\circ} \mathrm{C}$ & $106 \mathrm{~W} @-31.8^{\circ} \mathrm{C}$ & $7.9^{\circ} \mathrm{C}$ & & $86 \mathrm{~W} @-31.3^{\circ} \mathrm{C}$ & $7.8^{\circ} \mathrm{C}$ \\
\hline
\end{tabular}


两套流体回路在工作温区内的传热能力如图 6 所示，图中最下面的虚线是热控分系统对产品的传 热能力要求. 试验测得 1 \#回路(正常充装量)的传热能 力在 $-40^{\circ} \mathrm{C}$ 以上温区是明显大于 $2 \#$ 回路(少充装量), 这种能力差别在低温下不明显, 随着温度上升变得 明显. 但是, 两套回路的传热能力均满足要求.

对于 2 \#回路而言, 即使是驱动高度留有 $20 \mathrm{~mm}$ 余量, 其测得的传热能力仍然大于技术要求, 且有一 定的余量，这部分余量是两相回路的设计余量.

两套回路的系统传热温差如图 7 所示.

在各测试工作温度下，两套系统的传热温差均 小于 $10^{\circ} \mathrm{C}$, 满足技术要求的规定. 且在低温区, 如在 $-50^{\circ} \mathrm{C}$ 温度下, 1 \#回路的传热温差要明显小于 $2 \#$ 回 路的温差，可见足够的驱动力有利于减小系统传热

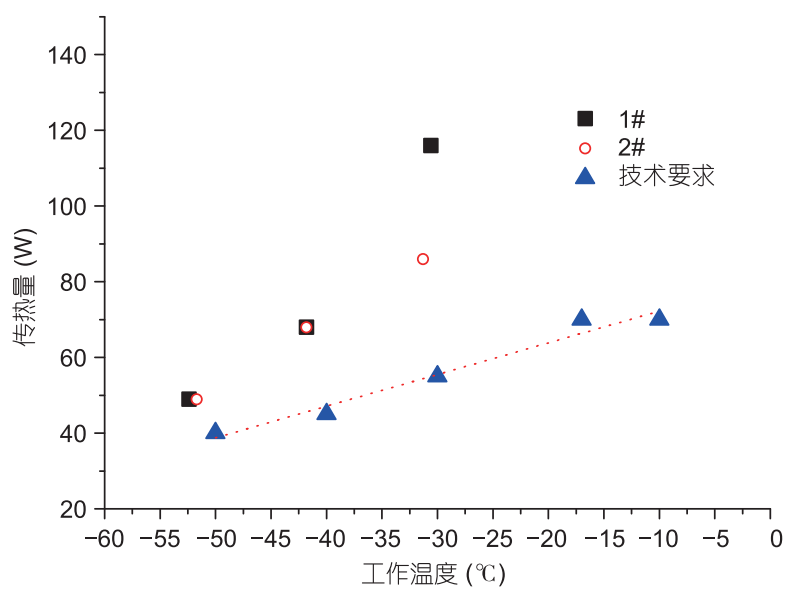

图 6 (网络版彩图)全温区传热能力曲线比较

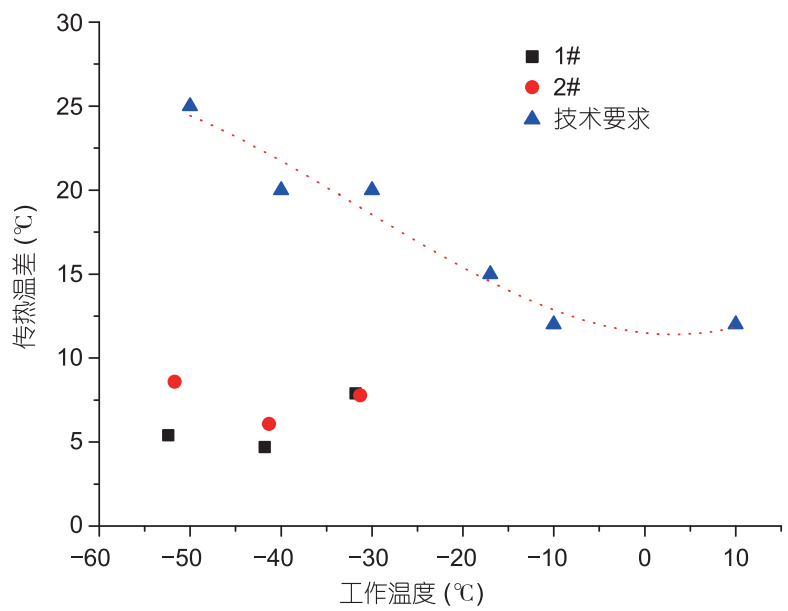

图 7 (网络版彩图)全温区传热温差曲线比较
温差. 这是因为, 对于两套回路, 系统的温差均主 要是蒸发器内的蒸气过热引起的, 而大的驱动力有 利于毛细结构对蒸发器的供液, 保持蒸发器内的含 液率.

\section{2 传热极限下的运行特性}

1) 传热极限

对于 $1 \#$ 回路, $-50^{\circ} \mathrm{C}$ 工况下, 在传热温差 $5.1^{\circ} \mathrm{C}$ 下 的传热能力为 $49 \mathrm{~W}$ (模拟热源 $50 \mathrm{~W}$, 系统漏热量 1 W). 试验中, 继续增大模拟热源的功率, 温度曲线如 图 8 所示, 当功率增大至 $60 \mathrm{~W}$ 时, 回路传热温差超 过 $10^{\circ} \mathrm{C}$, 达到 $15.1^{\circ} \mathrm{C}$; 当功率增加到 $70 \mathrm{~W}$ 时, 回路 仍能正常工作，但传热温差增大至 $17.4^{\circ} \mathrm{C}$.

传热温差增大的原因主要包括两个方面：一方 面, 由于蒸发器自身结构换热热阻的存在, 随着功率 增大，其传热温差增大；另一方面，随着功率的增大， 供液量需求增大, 毛细结构对蒸发器的供液阻力增 大, 供液相对不再充分, 从而导致蒸发器上部的过热 度增大，引起传热性能恶化。

当模拟热源功率继续增大时, 出现了蒸发器温 度陡升、回路失效的现象. 如图 9 所示, 初始段回路 工作在 $70 \mathrm{~W}$ 功率下，当模拟热源施加 $90 \mathrm{~W}$ 功率时, 蒸发器温度出现了陡升, 冷凝器和储液器温度下降, 蒸发器和储液器之间的温差短时间内增大至 $60^{\circ} \mathrm{C}$ 以 上，且蒸发器温度测点无平衡趋势，可见，即使容忍 巨大的传热温差, $90 \mathrm{~W}$ 也已经超出了 $-50^{\circ} \mathrm{C}$ 下流体回 路的传热极限。

停止加热并经过降温后，再次对模拟热源施加

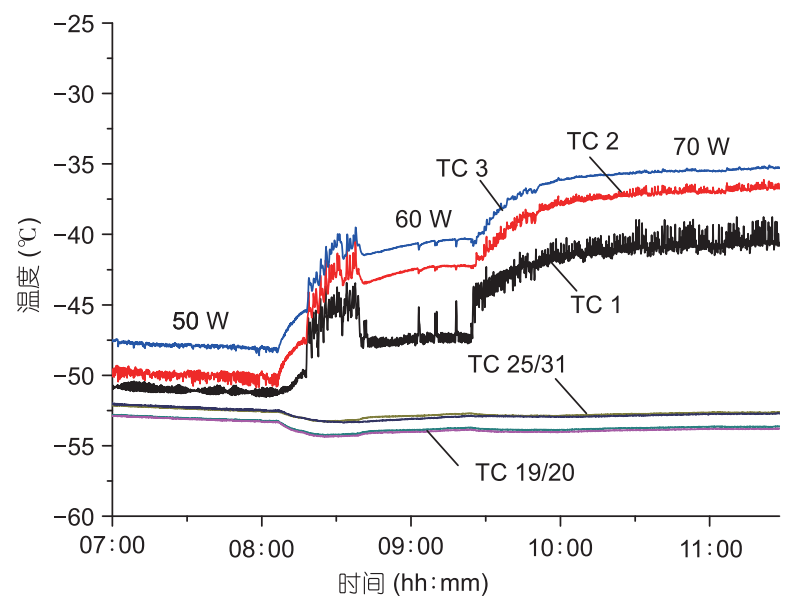

图 8 (网络版彩图) $-50^{\circ} \mathrm{C}$ 时传热能力测试工况(功率 $\mathbf{7 0} \mathrm{W}$ ) 


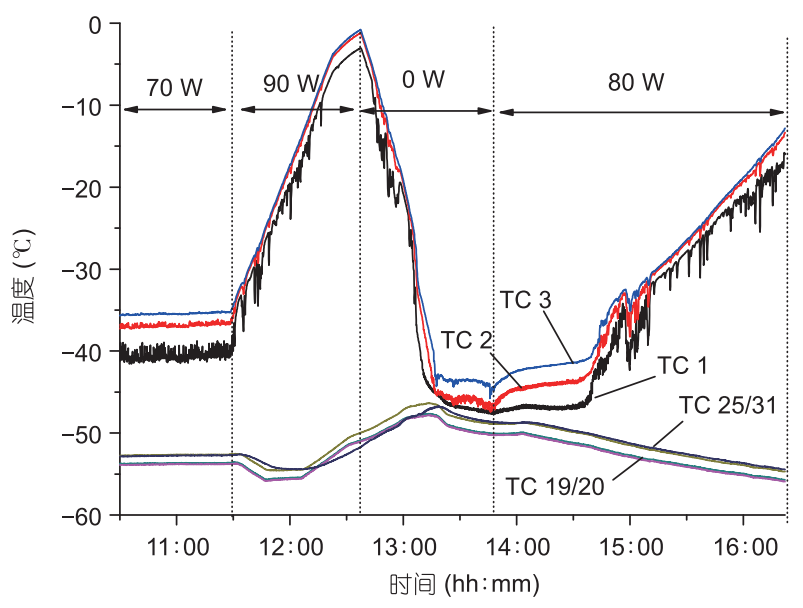

图 9 (网络版彩图) $-50^{\circ} \mathrm{C}$ 时传热能力测试工况(功率 $90 \mathrm{~W}$ 和 $80 \mathrm{~W}$ )

$80 \mathrm{~W}$ 功率, 失效现象再次出现, 不同于 $90 \mathrm{~W}$ 失效现 象的是这次温度陡升在施加功率后近 $1 \mathrm{~h}$ 才发生.

2) 亚稳定运行状态

对于两相系统, 其运行稳定性是工程应用中需 考虑的重要因素, 两相流体回路试验中也观察到过 一些亚稳定运行状态, 但是这种表现为温度波动的 状态并未发散而导致回路失效.

图 10 为 2\#回路在试验中出现的温度波动曲线图. 从 03:00 时刻开始, 对模拟热源突然直接施加 50 W 的功率, 蒸发器测点 $1 \#, 2 \#, 3 \#$ 温度值陡升, $1 \mathrm{~h}$ 后蒸 发器温度升到最大值, 随后开始减小, 从约 4:15 开 始烝发器温度测点 1\#, 2\#, 3\#, 25\#出现有规律的波动, 波动周期约 $3 \mathrm{~h}$, 回路运行仍然稳定.

此时回路虽然出现了温度波动现象, 但是仍然 能维持在一定温度范围内, 温度波动幅度不超过 $4^{\circ} \mathrm{C}$, 蒸发器和冷凝器的温差未持续上升, 回路并未失效, 处于一种亚稳定的运行状态.

产生两相系统不稳定现象的原因较复杂, 其内 部毛细供液影响、气泡的产生和消除、压力和温度的 变化等因素均可能导致两相流动、换热的不稳定问题, 直观的表现形式就是温度波动. 但是, 对于本系统而 言, 所有试验过程中, 这种温度波动仅出现在 $-50^{\circ} \mathrm{C}$

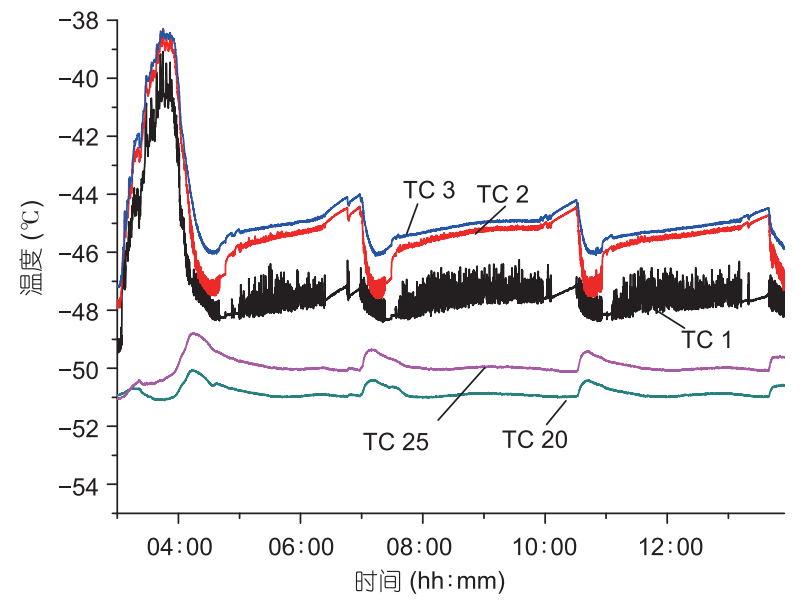

图 10 (网络版彩图) $-\mathbf{5 0}{ }^{\circ} \mathrm{C}$ 时传热能力测试工况(功率 $\mathbf{5 0} \mathrm{W}$ )

的低温、功率接近回路传热能力极限的工况下, 可见, 低温下回路的阻力接近其驱动力极限的情况下, 其 稳定性较差, 受干扰因素影响的机会较大.

\section{7 结论}

1) 建立了针对月球表面 $g / 6$ 重力驱动两相流体 回路系统的地面保守模拟试验验证方法, 满足了工 程应用的需要. 通过嫦娥三号着陆器和巡视器在月 面的多个月夜实际运行后的设备状态和遥测数据表 明, 两相流体回路运行良好, 满足了总体性能指标要 求，证明了该系统设计、试验方法合理、可行.

2) 采用等效验证方法, 对两套不同充装量的产 品进行真空热性能试验. 试验结果表明两套回路在 $-50^{\circ} \mathrm{C},-40^{\circ} \mathrm{C}$ 和 $-30^{\circ} \mathrm{C}$ 下具有较好的传热性能, 其测 试得出的传热能力和传热温差均能满足分系统提出 的研制技术要求.

3) 分析了两相流体回路的失效过程和现象, 回 路在接近传热能力极限时, 传热温差会随着传热量 增大而逐步增大, 直至失效. 在低温下、接近传热极 限时, 回路还可能会出现周期性的温度波动, 这种亚 稳定的运行状态会一直持续保持, 且并不会导致蒸 发器烧干.

\section{参考文献}

1 Zhao J F. Two-phase flow and pool boiling heat transfer in microgravity. Int J Multiphase Flow, 2010, 36: 135-143

2 Hepper D B, King C D, Littles J W. Zero-g experiments in two-phase fluid flow regimes. ASME Paper A75-40880, 1975

3 Zhao L, Rezkallah K S. Pressure drop in gas-liquid flow at microgravity conditions. Int J Multiphase Flow, 1995, 21: 837-849 
4 Kamiel S G. Microgravity Two-phase Flow and Heat Transfer. El Segundo: Springer Netherlands, 2007. 29-76

5 Colin C, Fabre J A, Dukler A E. Gas-liquid flow at microgravity conditions. Int J Multiphase Flow, 1991, 17: 533-544

\title{
Ground test method and results of closed two-phase thermosyphons for the moon exploration spacecraft Chang'E-3
}

\author{
ZHANG HongXing, MIAO JianYin, WANG Lu, MO Qing, LIU ZiJun \& XIANG YanChao \\ Beijing Key Laboratory of Space Thermal Control Technology, Institute of Spacecraft System Engineering, China Academy of Space Technology, \\ Beijing 100094, China
}

Closed two-phase thermosyphons were developed for the thermal control system in the Chinese moon exploration spacecraft Chang'E-3. This is the first space application for the thermosyphon. The biggest challenge of the thermal control system was to maintain the equipment temperature levels to help the lander and the rover survive the 14-earth day cold night without power supply. Closed two-phase thermosyphons were designed to transfer the RHU heat to the equipments at lunar night. The thermal control system could require no power supply and less weight to survive the explorer at lunar night. The ground test method was established to verify the heat transfer capability of the loops that will operate on the moon surface by sextupling the cross section area of the reservoir and decreasing the height difference between the reservoir and the reservoir to be a sixth of the real difference. The thermal vacuum test results of the two-phase loop designed for the lunar rover will be presented.

\section{thermosyphon, Chang'E-3, groud test method}

doi: 10.1360/092014-55 